January 2022

\title{
Adaptive Staffing Models and their Translation to Future Disaster Response: What Can Be Learned from COVID Unit Staffing?
}

LaKeisha Day PA-C

A.T. Still University, LaKeishaDay@atsu.edu

Follow this and additional works at: https://nsuworks.nova.edu/ijahsp

Part of the Health and Medical Administration Commons

\section{Recommended Citation}

Day L. Adaptive Staffing Models and their Translation to Future Disaster Response: What Can Be Learned from COVID Unit Staffing?. The Internet Journal of Allied Health Sciences and Practice. 2022 Jan 03;20(1), Article 16.

This Opinion Piece is brought to you for free and open access by the College of Health Care Sciences at NSUWorks. It has been accepted for inclusion in Internet Journal of Allied Health Sciences and Practice by an authorized editor of NSUWorks. For more information, please contact nsuworks@nova.edu. 


\title{
Adaptive Staffing Models and their Translation to Future Disaster Response: What Can Be Learned from COVID Unit Staffing?
}

\begin{abstract}
Background: As much as hospital organizations like to be prepared for worst-case scenarios, situations inevitably arise that are unanticipated for which no amount of preparation would ever suffice. One such scenario was the emergence of severe acute respiratory syndrome coronavirus 2 (SARS-CoV-2), the virus responsible for coronavirus disease 2019 (COVID-19). Hospitals had to use their existing staff in order to meet the onslaught of patients presenting with COVID-19. Purpose: The aim of this paper is to review the various COVID unit hospital staffing models that were implemented during the influx of patients with COVID-19 and then to examine their sustainability and adaptability to response models for future disasters. Hospitals are equipped with rapid response teams and some with disaster response teams, but neither is designed to be on a massive scale or for an extended duration. Which teams worked well, and can any be used in future disaster response staffing models? Conclusions: An adaptive and sustainable disaster response team is more about the members who make up the team, than it is about the response. The training and knowledge of health care providers determines staffing model adaptability and these characteristics can be targeted when making decisions on hiring, training, ongoing education, and staff assignments.
\end{abstract}

\section{Author Bio(s)}

LaKeisha Day, PA-C, CMQ has served as the Advanced Practice Provider Supervisor for the Nocturnal Program at the University of Texas, MD Anderson Cancer Center for the past eight years. Presently, she is pursuing a Doctor of Medical Science Degree at A.T. Still University, Arizona School of Health Sciences. She is also certified in Medical Quality.

\section{Acknowledgements}

Dr. Randy Danielsen, Professor Medical Writing, ATSU Anayo Mbadugha, PA-C, colleague and supporter 


\title{
IIIAHSP \\ The Internet Joumnal of Allied Health Sciences and Practice \\ Dedicated to allied health professional practice and education \\ Vol. 20 No. 1 ISSN 1540-580X
}

\section{Adaptive Staffing Models and their Translation to Future Disaster Response: What Can Be Learned from COVID Unit Staffing?}

\author{
LaKeisha Day
}

A.T. Still University

United States

\begin{abstract}
Background: As much as hospital organizations like to be prepared for worst-case scenarios, situations inevitable arise that are unanticipated for which no amount of preparation would ever suffice. One such scenario was the emergence of severe acute respiratory syndrome coronavirus 2 (SARS-CoV-2), the virus responsible for coronavirus disease 2019 (COVID-19). Hospitals had to use their existing staff in order to meet the onslaught of patients presenting with COVID-19. Purpose: The aim of this paper is to review the various COVID unit hospital staffing models that were implemented during the influx of patients with COVID-19 and then to examine their sustainability and adaptability to response models for future disasters. Hospitals are equipped with rapid response teams and some with disaster response teams, but neither is designed to be on a massive scale or for an extended duration. Which teams worked well, and can any be used in future disaster response staffing models?

Conclusions: An adaptive and sustainable disaster response team is more about the members who make up the team, than it is about the response. The training and knowledge of health care providers determines staffing model adaptability and these characteristics can be targeted when making decisions on hiring, training, ongoing education, and staff assignments.
\end{abstract}

Keywords: COVID unit, staffing models, disaster response 


\section{INTRODUCTION}

Health care workers are resilient, and their resiliency is tested every day. The ultimate test came in the spring of 2020; when society came to a halt, and the roles and responsibilities of health care and other essential workers expanded exponentially. For hospital workers who immediately began to care for patients with coronavirus disease of 2019 (COVID-19), it was not only a test in resiliency, but also in character, patience, and adaptability. President and CEO of Atrium Health, Eugene Woods, when describing their hospital system's pandemic response efforts, stated "When it matters most, the best of who we are shows up."1(p94) Hospital operations evolved to facilitate the care for a new type of patient, when testing was still not readily available and the expected disease course was still relatively unknown. Likewise, patient care providers made changes to their workflows and schedules, and in the process, acquired new skills and knowledge as the months of the pandemic continued. By nature, disaster response is unanticipated, and the deployment of resources is based on the characteristics of the existing workforce. There are many examples of how different hospitals and teams demonstrated resourcefulness and leveraged their existing workforce to meet the need. Among these examples are many approaches that could be reproducible for future disaster response staffing models. Although some may not be able to be implemented entirely, even the adaptation of some aspects could provide a framework that clinicians involved in inpatient hospital operations and medical practice can use.

\section{REVIEW OF THE LITERATURE}

Some hospitals have provided a myriad of experiences with COVID unit staffing; including perspectives from ICU physicians, hospitalists, clinical nurses, respiratory therapists, and dietitians to name a few. These perspectives show how their existing workforce was used to mount an initial response. While most of the literature details COVID intensive care unit (ICU) staffing, many of the methods used could be adapted to medical units for other disaster response operations. "Health care leaders are hopeful that lessons learned during the pandemic will prove helpful in addressing workforce shortages that existed before the health crisis and are expected to worsen as millions age out of the job market."2(p17)

During the height of the pandemic, the New York-Presbyterian (NYP) hospital system demonstrated adaptability and resiliency by not only increasing ICU bed capacity but also by harnessing the full potential of the existing workforce. It implemented dynamic staffing models that challenged traditional roles of health care providers. Their staffing mechanism was a tiered and team-based approach that was highlighted by the presence of a critical care attending physician in an oversight role for as many as 50 patients at a time. ${ }^{3}$ After analyzing the skills and knowledge of the providers on staff, teams were instituted based on critical care management capabilities as opposed to traditional roles based on title or professional designation. One example of a team in this dynamic staffing model was that of a team led by a cardiologist that was supported by pediatric physicians, residents from psychiatry, surgery, and physical medicine and rehab, skilled registered nurses, and respiratory therapists. When a concern was raised that more critical care expertise would be needed, a critical care nurse practitioner was added. With this model, there were instances when clinical fellows acted in the role of an attending physician and some advanced practice providers (APPs) supervised attendings. Consult teams (proning teams, intubation teams, procedure teams, etc) were implemented in order to provide advanced level skills support and to unburden providers from time-consuming tasks.

The largest public health system in the Unites States, New York City Public Health + Hospitals, acted rapidly in order to train, reassign, and redeploy its existing workforce, as well as to hire and onboard people for new positions to address the onslaught of patients during the surge. ${ }^{4}$ Their strategy involved the reassignment of a wide range of clinical providers from pediatricians to dentists in order to redesign patient care workflows and responsibilities to meet patient care needs. In some cases, the emergency department was staffed with internists, dentists were deployed as phlebotomists, and pediatricians worked in adult ICUs. A new role was also created in order to address the changing guidance and availability of personal protective equipment (PPE). This "PPE Officer" was a new position that was typically filled by an APP.

The University of Pittsburg Medical Center augmented its ICU staffing and implemented it across a 40-hospital system that spans 3 states. ${ }^{5} \mathrm{~A}$ comprehensive staffing model was designed that was rooted in the framework provided by existing guidelines from the Centers for Disease Control and Prevention, National Academies of Science, and the Society of Critical Care Medicine (SCCM). At the time, the existing literature addressed seasonal influenza outbreaks and mass casualty events, neither of which would encompass the scale and breadth of the COVID-19 outbreak. The hospital system's surge plan first identified and classified the existing staffing resources in the ICUs and organized them into different tiers based on their level of critical care expertise. Critical care providers were classified as the first tier, ICU-capable providers (including ICU capable APPs) were classified as second tier, and those without previous critical care experience were classified as third tier. There was also a "flex tier" for providers with more specialized skills sets such as intubation, patient proning, central and arterial line placement, etc. A surge capacity protocol was established and deployed the teams across the ICUs depending on patient volumes. With this model, a single experienced critical care physician ("tier 1") would oversee the care of up to 24 patients, either remotely or on-site. A highlight of their design was the 
strategic and deliberate placement of APPs throughout their tiered model. Much like the model established at the NYP hospital system, the APPs functioned as team leaders.

The University of California San Francisco took an approach that first sought to leverage the existing workforce resources, such as reassigning APPs and trainees from their assigned clinical duties and redeploying them into other areas in order to meet patient care needs. ${ }^{6}$ The hospital harnessed the "pluripotent" nature of hospitalists in order to manage the needs of this complex patient population. The clinical work of hospitalists was prioritized because of their inpatient experience. The hospital also sought to address long-term effects such as staff burnout and morale changes that resulted from the response mechanisms. Some frontline providers were given operational roles in addition to their clinical duties in order to streamline and disperse information to their teams.

\section{COMPREHENSIVE COVID COHORT STAFFING}

At the University of Texas MD Anderson Cancer Center the approach to staffing not only had to leverage the existing workforce but also deal with the additional challenge of keeping suspected or confirmed COVID patients separate from the existing immunocompromised inpatient population. With elective surgery cases halted, one floor that previously housed post-operative surgical patients was made available to house suspected and confirmed COVID patients, further isolated on two separate pods. At the peak of the response, these units expanded to the three floors directly below the original one. Initial provider staffing for the COVID units was accomplished on a mostly voluntary basis. Rigorous efforts were undertaken in order to have all the incoming COVID unit providers updated on personal protective equipment (PPE) training, granted disaster privileges, and educated on patient management algorithms.

Provider teams were divided into three groups based on patient classification: persons under investigation (PUIs), patients confirmed positive for COVID-19, and confirmed positive patients requiring intensive care. An example of the staffing for the COVID unit is shown in Table 1. The designation of a PUI was based on Centers for Disease Control (CDC) guidelines for symptomatic testing because at the time the unit opened, the testing took anywhere from 16 to 24 hours for results to be available. Early in the pandemic, the census of the PUI unit far outnumbered that of the two other COVID unit designations. Once more rapid testing became available, those numbers inverted.

\section{Table 1 COVID Unit Cohort Staffing}

\begin{tabular}{cccc} 
& \multicolumn{1}{c}{ PUI } & CONFIRMED COVID & COVID ICU \\
\hline DOSPITALIST SERVICE & 1APP, 1MD & $\begin{array}{c}\text { Variable APP/MD team } \\
\text { composition based on location } \\
\text { and census }\end{array}$ & $1 \mathrm{MD}$ \\
\hline CRITICAL CARE & & $1 \mathrm{APP}, 1 \mathrm{MD}$ \\
\hline NIGHT & 1 APP, 1MD & $\begin{array}{c}\text { Variable APP/MD team } \\
\text { composition based on location } \\
\text { and census }\end{array}$ & $1 \mathrm{MD}+/$ - APP \\
\hline NOCTURNAL PROGRAM & & & \\
\hline
\end{tabular}

*PUI: Persons under investigation, currently dissolved and PUIS are housed on floors outside of the COVID unit in negative pressure isolation rooms.

+Allencompassing program that provides overnight and weekend patient coverage to the entire hospital across all disciplines; consists of staff APPS and moonlighting MDs.

The provider composition of the workforce on the COVID units took on different characteristics as the hospital's operations evolved. Since elective operations were postponed and clinic appointments were rescheduled, the inpatient hospital census dropped significantly. This opened the opportunity to staff the units with providers from other disciplines such as surgeons, APPs, certified registered nurse anesthetists (CRNAs), and staff physicians from consultative services such as pulmonary medicine. Assignments were made based on voluntary availability and area of expertise. As one of the largest employers of APPs in the country, MD Anderson was able to easily and effectively incorporate APPs into all three COVID units around the clock. APPs and MDs worked synergistically in the units to provide comprehensive care along with valuable clinical guidance from the primary oncology teams, who provided care virtually.

The staffing was scaled up or down based on the census of the units, and many teams mobilized APPs and moonlighting physicians to achieve the necessary staffing. The nocturnal program stretched to meet the demand by increasing the pool of moonlighters to also include APPs from primary and consultant teams. For several months at the height of the surge one consultant team that is 
primarily staffed with APPs focused on blood management and provided staffing for the patients who required blood management at night, thus allowing a nocturnal APP who is usually assigned to this role to mobilize to the COVID unit.

\section{CORRELATION WITH DISASTER RESPONSE TEAMS, PRESENT AND FUTURE}

Hospital disaster response teams are prevalent around the country and typically are tailored to the population in the region and the hospital system. For the population of patients in hospitals, various rapid response systems, such as stroke and code blue teams exist. To address patient care that originates outside of the hospital, there are emergency response teams such as those in the emergency rooms (shock rooms) and trauma operating rooms. These responses have a short-term and limited scope and treat acutely ill patients. For patients requiring long-term management and resources, the response takes on a larger scale. Much like the COVID response teams, disaster response teams use the existing workforce in highly specialized roles. As one text on disaster response in hospitals noted, "It is not sufficient that healthcare organizations plan for a single event; rather, they must develop the capacity to respond to combinations of escalating events." ${ }^{\prime(p 7) ~ H o s p i t a l s ~ a r e ~ b e g i n n i n g ~ t o ~ h a r n e s s ~ t h e ~ f u l l ~ c a p a c i t y ~ o f ~ p a t i e n t ~}$ monitoring in order to deploy early warning teams for sepsis, cardiac events, and other indicators of clinical deterioration. These teams have specialized training that can be broadened to incorporate additional training in emergency and disaster response.

\section{DISCUSSION}

Disaster and emergency response teams use existing resources to address medical emergencies. Some features that can translate to future events include leveraging existing staff to the top of their skills, training the workforce in varying roles, and keeping roles in disaster protocols in mind when hiring. Having staff educated on patient management protocols and PPE requirements was common among the initial responses. The ubiquitous presence of the APP was found in all cases mentioned and could therefore provide an opportunity for future response planning and workforce modeling. The scope of training and capability of APPs is vast and can be used in various levels of patient management teams. Remembering the reliance on them in disaster response is instrumental in planning for the future. A recent article in the Journal of American Academy of Physician Assistants (JAAPA) also espoused the expanded role of APPs during the pandemic: "teams of PAs, NPs, and physicians can then gracefully navigate changing workforce needs due to COVID-19 surges, future pandemics, and other disruptions such as duty hour restrictions. ${ }^{8(p 3)}$

\section{CONCLUSION}

Surge response planning gave hospitals the blueprint for future disaster response staffing models that can be tailored to the existing characteristics of the workforce and can potentially be adapted as a first option for future disaster response staffing. Because of the unpredictability of such occurrences, hospitals cannot hire on the sole basis of an event potentially occurring. However, hospitals can take the examples of the inpatient teams that were formed around the world and start to consider how these teams can be best used when a response is warranted. While guidelines exist for disaster planning, having a system in place that is specific to an organization's needs will be paramount. It is important to learn the lessons from the current pandemic in order to maintain preparedness for the future. One solution could be to regularly train and credential a small number of staff in acute care medicine and disaster management, in addition to their regular job role. These individuals can then be deployed as an initial response in caring for a sudden onslaught of patients, and in the meantime provide hospital units with additional time to coordinate a subsequent response. An inclement weather or "ride out" team is another example of an existing disaster response mechanism used by hospitals to ensure adequate staffing when there is a concern that the workforce would not be able to safely travel to and from the workplace. A similar approach could also be considered when deploying a team to respond to disasters in the future. Having health care teams that are aware and prepared in advance for these roles could be an important factor in future disaster response.

\section{References}

1. Woods E. When it matters most, the best of who we are shows up. JHM. 2021;66(2):91-94. doi:10.1097/JHM-D-2100034

2. Van Dyke M. Leveraging your workforce: getting creative. Healthcare Executive. 2021;36(3):16-22

3. Anderson BR, Ivascu NS, Brodie D, et al. Breaking silos: the team-based approach to coronavirus disease 2019 pandemic staffing. Crit Care Explor. 2020;2(11):e0265. doi:10.1097/CCE.0000000000000265

4. Keeley C, Jimenez J, Jackson $\mathrm{H}$, et al. Staffing up for the surge: expanding the New York City Public Hospital Workforce during the COVID-19 Pandemic. Health Affairs. 2020;39(8):1426-1430. doi:10.1377/hlthaff.2020.00904

5. Harris GH, Baldisseri MR, Reynolds BR, Orsino AS, Sackrowitz R, Bishop JM. Design for implementation of a systemlevel icu pandemic surge staffing plan. Crit Care Explor. 2020;2(6):e0136. doi:10.1097/CCE.0000000000000136

6. Garg M, Wray CM. Hospital medicine management in the time of covid-19: preparing for a sprint and a marathon. J Hosp Med. 2020;15(2020-05):305-307. doi:10.12788/jhm.3427 
7. Cetaruk E, Birou D, Glarum JF. Hospital Emergency Response Teams: Triage for Optimal Disaster Response. Elsevier Science Limited; 2010.

8. McGrath, Bridget A. PA-C; Saltzman, Jina PA-C; Cardin, Tracy APN-C; Callender, Brian MD Modernizing the PA and NP workforce. Journal of the American Academy of Physician Assistants. 2021;34(8):1-3. doi:

10.1097/01.JAA.0000753924.93183.e 